\title{
Free Vibrations of Thin Shells with Isogeometric Approach
}

\author{
Sang Jin LEE \\ ADOPT Research Group, Department of Architectural Engineering, Gyeongsang National University
}

http://dx.doi.org/10.5659/AIKAR.2014.16.2.67

\begin{abstract}
Free vibration analysis of thin shells is carried out by using isogeometric approach. For this purpose, a thin shell element based on Kirchhoff-Love shell theory is developed. Non-uniform rational B-spline surface (NURBS) definition is introduced to represent the geometry of shell and also used to derive all terms required in the isogeometric element formulation. Gauss integration rule is used for stiffness and mass matrices. The present shell element is then applied to examine vibrational behaviours of thin plate and shell structures. From numerical results, it is found be that reliable natural frequencies and associated mode shapes of thin shell structures can be predicted by the present isogeometric shell element.
\end{abstract}

Keywords: Thin Shell, Isogeometric Analysis, Free Vibration, B-spline, NURBS.

\section{INTRODUCTION}

There is prominent importance to understand the vibrational behaviours of shells in structural design. So far, many attempts have been made to calculate the accurate natural frequencies and associated mode shapes of shells. The first attempt was made by Love (1888) and Rayleigh (1894) and then Flügge (1934) provided numerical solution of shell vibrations using frequency equation. With the introduction of numerical analysis techniques (Leissa, 1973; Qatu, 1992; Liew, 1995), various types of shells with complex geometry have been gradually tackled. In particular, with the use of FE technologies, thin shells tend to be more widely used in various engineering practices and therefore vibrations of thin shells become more crucial. In recent year, as an alternative method to the FE method, the isogeometric analysis (IGA) concept has been proposed by Hughes et al. (2005). In the isogeometric approach, NURBS (De Boor, 1978) is consistently used to represent both structural geometry and the displacement field. As well-known fact, the NURBS can provide higher continuity of derivatives compared to the interpolation functions such as Lagrange interpolation function. The NURBS basis functions can be also efficiently refined without changing the geometry (Cottrell et al., 2009) and the order of the basis function can be naturally elevated. There have been several applications of isogeometric concept into structural vibrational problem (Hughes and Evans, 2010). In particular,

Corresponding Author: Sang Jin LEE, Professor

ADOPT Research Group, Department of Architectural Engineering,

Gyeongsang National University, Jinju, Korea

Tel : +82-55-772-1754 e-mail : lee@gnu.ac.kr

This is an Open Access article distributed under the terms of the Creative Commons Attribution Non-Commercial License (http://creativecommons. org/licenses/by-nc/3.0/) which permits unrestricted non-commercial use, distribution, and reproduction in any medium, provided the original work is properly cited. the detailed investigations on the vibrational behaviours of plate and Timoshenko beam are provided by Lee and Kim (2012) and Lee and Park (2013) respectively. As a sequel of the previous study on the vibrations of beams and plates, we here introduce the isogeometric approach to investigate natural frequencies and associated mode shapes of thin shells. For this purpose, a thin shell element based on Kirchhoff-Love shell theory is developed with isogeometric concept. Hence, NURBS definition is introduced to represent the geometry of shell and also used to derive all terms required in the isogeometric element formulation. In addition, Gauss integration rules is used for stiffness and mass matrices. Some benchmark tests are carried out and provided as the future reference solution based on isogeometric approach for thin plate and shell structures.

\section{B-SPLINES}

\subsection{Knot vector}

A knot vector $\boldsymbol{\Xi}$ is a set of non-decreasing real values that constitutes a set of coordinates in the parametric space:

$$
\Xi=\left[\xi_{1}, \xi_{2}, \xi_{3}, \ldots, \xi_{n+p+1}\right]
$$

where $n$ is the number of basis functions and $p$ is the order of the B-spline. A knot vector is said to be uniform if its knots are uniformly spaced and non-uniform otherwise. Moreover, a knot vector is said to be open if its first and last knots are repeated $p+1$ times. Basis functions formed from open knot vectors are interpolatory at the ends of the parametric interval $\left[\xi_{1}, \xi_{n+p+1}\right]$ but are not, in general, interpolatory at interior knots. It should be noted that we would employ open knot vectors throughout the analysis of laminated plate.

\subsection{Basis functions}

B-spline basis function (De Boor, 1978) is defined recursively 


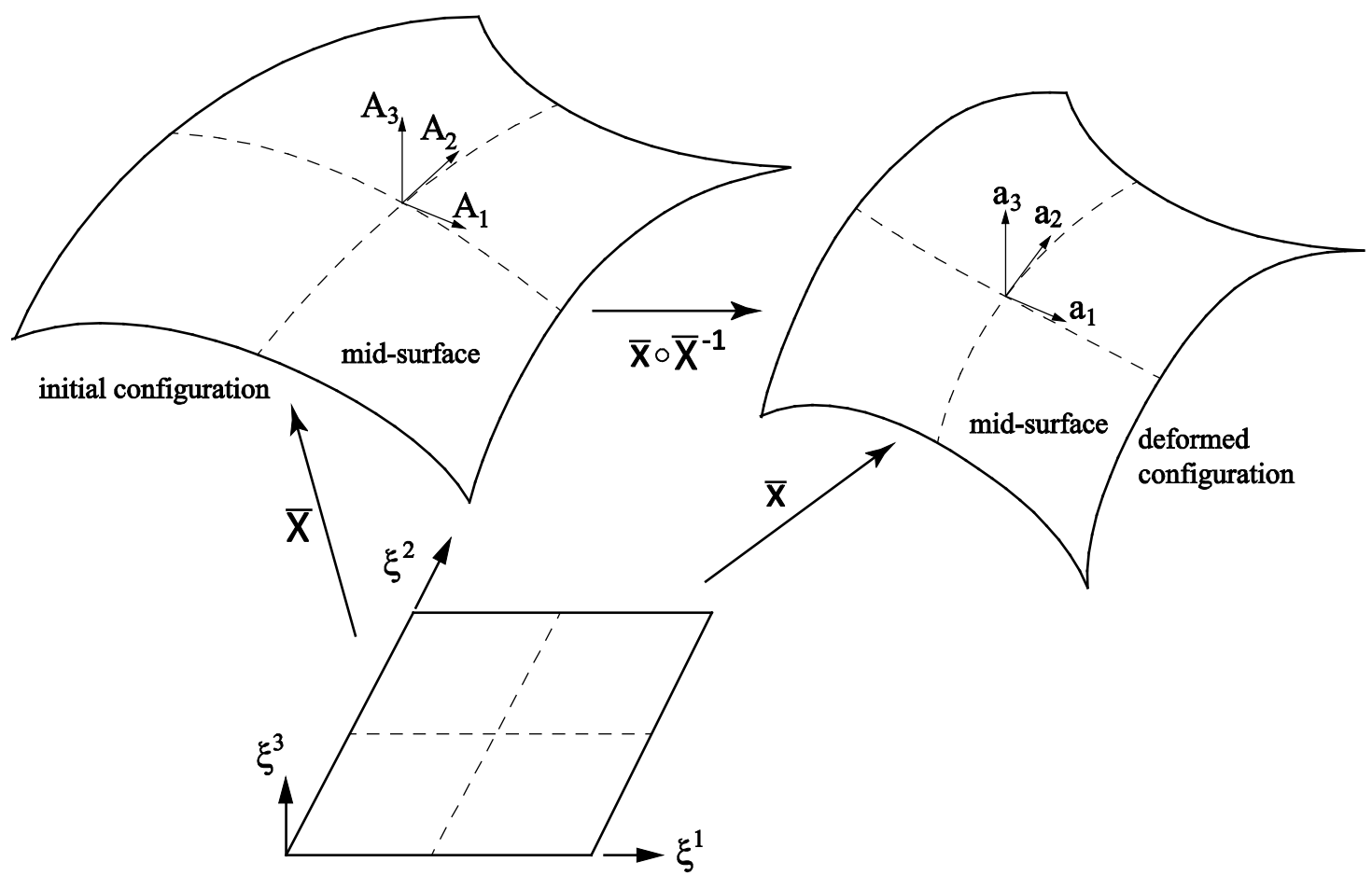

Figure 1. Shell mid-surface configurations with parameterizations

starting with $\mathrm{p}=0$ as:

$$
N_{i, 0}(\xi)=\left\{\begin{array}{lr}
1 & \text { if } \xi_{i} \leq \xi<\xi_{i+1} \\
0 & \text { otherwise }
\end{array}\right.
$$

For $\mathrm{p} \geq 1$

$$
\begin{aligned}
N_{i, p}(\xi)= & \frac{\xi-\xi_{i}}{\xi_{i+p}-\xi_{i}} N_{i, p-1}(\xi) \\
& +\frac{\xi_{i+p+1}-\xi}{\xi_{i+p+1}-\xi_{i+1}} N_{i+1, p-1}(\xi) .
\end{aligned}
$$

\subsection{B-spline curves}

We construct basis functions with the order of a B-spline and an appropriately defined knot vector. The piecewise polynomial B-spline curve $S(\xi)$ of order $p$ can be obtained by taking a linear combination of basis function and control points:

$$
S(\xi)=\sum_{i=1}^{n} N_{i, p}(\xi) C_{i}
$$

where $\mathrm{C}_{i}$ is the $i^{\text {th }}$ control point. The piecewise linear inter-polation of the control points defines the control net.

\subsection{B-spline surface}

We construct a B-spline surface using tensor product of spline curve in the direction of $\xi$ and $\eta$ :

$$
\begin{aligned}
S(\xi, \eta) & =\sum_{i=1}^{n} \sum_{j=1}^{m} N_{i, p}(\xi) N_{j, q}(\eta) C_{i, j} \\
& =\sum_{i=1}^{n} \sum_{j=1}^{m} R_{i, j}^{p, q}(\xi, \eta) C_{i, j} .
\end{aligned}
$$

\subsection{NURBS}

NURBS can be defined as

$$
S(\xi, \eta)=\sum_{i=1}^{n} \sum_{j=1}^{m} R_{i, j}^{p, q}(\xi, \eta) C_{i, j}
$$

where $R_{i, j}^{p, q}$ can be written as

$$
R_{i, j}^{p, q}(\xi, \eta)=\frac{N_{i, p}(\xi) N_{j, q}(\eta) w_{i, j}}{\sum_{i=1}^{n} \sum_{j=1}^{m} N_{i, p}(\xi) N_{j, q}(\eta) w_{i, j}}
$$

and $w_{i, j}$ is the weight associated with the control point.

\section{KIRCHHOFF-LOVE SHELL THEORY}

\subsection{Assumptions}

The present isogeometric shell element is based on KirchhoffLove assumptions:

[A1]. All points lying on the normal of the mid-surface before deformation remain on that normal, which remains the normal of the deformed mid-surface.

[A2]. The distance of a point from the mid-surface remains constant during deformation.

Assumption [A1] is known as the Bernoulli hypothesis in the beam theory and therefore deformations due to transverse shear stresses are negligible. From assumption [A2], strain in normal direction is of no significance so that three-dimensional problem can reduce to a two dimensional surface deformation problem. 


\subsection{Kinematics}

The configurations of shell are described by the mid-surface of shell as illustrated in Figure 1 and the shell mid-surfaces are represented by using natural coordinate system $\left(\xi^{i}\right)$ such as

$$
\overline{\mathbf{x}}\left(\xi^{1}, \xi^{2}\right) \text { and } \overline{\mathbf{x}}\left(\xi^{1}, \xi^{2}\right)
$$

The initial geometry of shell is represented by the following expression:

$$
\mathbf{X}\left(\xi^{1}, \xi^{2}, \xi^{3}\right)=\overline{\mathbf{X}}\left(\xi^{1}, \xi^{2}\right)+\xi_{3} \mathbf{A}_{3}\left(\xi^{1}, \xi^{2}\right)
$$

where $\overline{\mathbf{X}}$ is the mid-surface of the shell and $\mathbf{A}_{3}$ is the normal to the mid-surface such as

$$
\mathbf{A}_{3}=\frac{\mathbf{A}_{1} \times \mathbf{A}_{2}}{\left|\mathbf{A}_{1} \times \mathbf{A}_{2}\right|}
$$

in which $\mathbf{A}_{\alpha}=\overline{\mathbf{X}}_{, \alpha}(\alpha \in\{1,2\})$ is tangent vectors on the midsurface.

The deformed shell can be similarly defined as

$$
\mathbf{x}=\overline{\mathbf{x}}\left(\xi^{1}, \xi^{2}\right)+\xi^{3} \mathbf{a}_{3}
$$

where $\overline{\mathbf{x}}$ is the mid-surface of deformed shell and it can be written as

$$
\overline{\mathbf{x}}\left(\xi^{1}, \xi^{2}\right)=\overline{\mathbf{X}}\left(\xi^{1}, \xi^{2}\right)+\mathbf{u}\left(\xi^{1}, \xi^{2}\right)
$$

in which $\mathbf{u}$ is the displacement field of shell and $\mathbf{a}_{3}$ is the normal to the deformed mid-surface

The covariant base vectors in the initial and the deformed configurations are

$$
\begin{aligned}
& \mathbf{G}_{\alpha}=\frac{\partial \mathbf{X}}{\partial \xi_{\alpha}}=\mathbf{A}_{\alpha}+\xi^{3} \mathbf{A}_{3, \alpha} \\
& \mathbf{g}_{\alpha}=\frac{\partial \mathbf{x}}{\partial \xi_{\alpha}}=\mathbf{a}_{\alpha}+\xi^{3} \mathbf{a}_{3, \alpha} .
\end{aligned}
$$

The corresponding covariant components of the metric tensors in both configurations are

$$
\mathrm{G}_{\alpha \beta}=\mathbf{G}_{\alpha} \cdot \mathbf{G}_{\beta} ; \mathbf{g}_{\alpha \beta}=\mathbf{g}_{\alpha} \cdot \mathbf{g}_{\beta} .
$$

\subsection{Strain}

For measuring the deformation of shell, the Green-Lagrange strain tensor is used:

$$
\varepsilon_{\alpha \beta}=\frac{1}{2}\left(\mathrm{~g}_{\alpha \beta}-\mathrm{G}_{\alpha \beta}\right) .
$$

The linearization of (14) will be written as

$$
\varepsilon_{\alpha \beta}=\bar{\varepsilon}_{\alpha \beta}+\xi^{3} \kappa_{\alpha \beta}
$$

where the membrane and bending strain tensor are

$$
\begin{aligned}
\bar{\varepsilon}_{\alpha \beta} & =\frac{1}{2}\left(\mathbf{a}_{\alpha} \mathbf{a}_{\beta}-\mathbf{A}_{\alpha} \mathbf{A}_{\beta}\right) \\
\kappa_{\alpha \beta} & =\left(\mathbf{a}_{\alpha} \mathbf{a}_{3, \beta}-\mathbf{A}_{\alpha} \mathbf{A}_{3, \beta}\right) .
\end{aligned}
$$

Using (11), the above equation can be written as

$$
\begin{aligned}
\bar{\varepsilon}_{\alpha \beta} & =\frac{1}{2}\left(\mathbf{A}_{\alpha} \mathbf{u}_{, \beta}+\mathbf{A}_{\beta} \mathbf{u}_{, \alpha}+\mathbf{u}_{, \alpha} \mathbf{u}_{, \beta}\right) \\
\kappa_{\alpha \beta} & =\left(\mathbf{A}_{\alpha, \beta} \mathbf{A}_{3}-\mathbf{A}_{\alpha, \beta} \mathbf{a}_{3}-\mathbf{u}_{, \alpha \beta} \mathbf{a}_{3}\right) .
\end{aligned}
$$

For small deformation, membrane strain is reduced into the followings

$$
\begin{gathered}
\bar{\varepsilon}_{\alpha \beta}=\frac{1}{2}\left(\mathbf{A}_{\alpha} \mathbf{u}_{, \beta}+\mathbf{A}_{\beta} \mathbf{u}_{, \alpha}\right) \\
\kappa_{\alpha \beta}=-\mathbf{u}_{, \alpha \beta} \mathbf{A}_{3}+\frac{1}{\sqrt{a}}\left(\mathbf{u}_{, 1} \cdot\left(\mathbf{A}_{\alpha, \beta} \times \mathbf{A}_{2}\right)+\mathbf{u}_{, 2} \cdot\left(\mathbf{A}_{1} \times \mathbf{A}_{\alpha, \beta}\right)\right) \\
+\frac{\mathbf{A}_{3} \times \mathbf{A}_{\alpha, \beta}}{\sqrt{a}}\left(\mathbf{u}_{, 1} \cdot\left(\mathbf{A}_{2} \times \mathbf{A}_{3}\right)+\mathbf{u}_{, 2} \cdot\left(\mathbf{A}_{3} \times \mathbf{A}_{1}\right)\right)
\end{gathered}
$$

\subsection{Strain energy}

If the shell is linear elastic, the strain energy density can be written as

$$
\begin{aligned}
\mathrm{W}(\alpha, \beta)=\frac{1}{2}\left(\frac{E h}{1-v^{2}} H^{\alpha \beta \gamma \delta} \varepsilon_{\alpha \beta} \varepsilon_{\gamma \delta}\right. \\
\left.+\frac{E h^{3}}{12\left(1-v^{2}\right)} H^{\alpha \beta \gamma \delta} \kappa_{\alpha \beta} \kappa_{\gamma \delta}\right)
\end{aligned}
$$

where $\mathrm{E}$ is Young' modulus, $v$ is Poisson's ratio and

$$
H^{\alpha \beta \gamma \delta}=v A^{\alpha \beta} A^{\gamma \delta}+\frac{1}{2}(1-v)\left(A^{\alpha \gamma} A^{\beta \delta}+A^{\alpha \delta} A^{\beta \gamma}\right)
$$

where the contravariant components of the surface metric tensor

$$
A^{\alpha \delta} A_{\delta \beta}=\delta_{\beta}^{\alpha}
$$

in which $\delta_{\beta}^{\alpha}$ is Kronecker delta and covariant metric tensor is given by

$$
A_{\alpha \beta}=\mathbf{A}_{\alpha} \cdot \mathbf{A}_{\beta} .
$$




\subsection{Stress resultants}

The stress resultants from membrane and bending energies will be

$$
\begin{gathered}
n^{\alpha \beta}=\frac{\partial W}{\partial E_{\alpha \beta}}=\frac{E h}{1-v^{2}} H^{\alpha \beta \gamma \delta} \varepsilon_{\gamma \delta} \\
m^{\alpha \beta}=\frac{\partial W}{\partial \mathrm{K}_{\alpha \beta}}=\frac{E h^{3}}{12\left(1-v^{2}\right)} H^{\alpha \beta \gamma \delta} \kappa_{\gamma \delta} .
\end{gathered}
$$

Explicitly, the above stress resultant matrix can be written as

$$
\begin{gathered}
\left\{\begin{array}{l}
n^{\alpha \beta} \\
n^{\alpha \beta} \\
n^{\alpha \beta}
\end{array}\right\}=\frac{E h}{1-v^{2}} \widetilde{\mathbf{D}}\left\{\begin{array}{l}
\varepsilon_{\gamma \delta} \\
\varepsilon_{\gamma \delta} \\
\varepsilon_{\gamma \delta}
\end{array}\right\} \\
\left\{\begin{array}{l}
m^{\alpha \beta} \\
m^{\alpha \beta} \\
m^{\alpha \beta}
\end{array}\right\}=\frac{E h^{3}}{12\left(1-v^{2}\right)} \widetilde{\mathbf{D}}\left\{\begin{array}{l}
\kappa_{\gamma \delta} \\
\kappa_{\gamma \delta} \\
\kappa_{\gamma \delta}
\end{array}\right\}
\end{gathered}
$$

where the rigidity matrix $\widetilde{\mathbf{D}}$ is

$$
\begin{aligned}
& \widetilde{\mathbf{D}}= \\
& {\left[\begin{array}{ccc}
A_{11}^{2} & v A_{11} A_{22}+(1-v) A_{12}^{2} & A_{11} A_{12} \\
& A_{22}^{2} & A_{22} A_{12} \\
\text { sym. } & & (1-v) A_{11} A_{22}+(1+v) A_{12}^{2}
\end{array}\right] .}
\end{aligned}
$$

The stress resultant can be rewritten as

$$
\mathbf{n}=\mathbf{A} \varepsilon ; \mathbf{m}=\mathbf{D} \boldsymbol{\kappa}
$$

where the vectors $\mathbf{n}, \mathbf{m}$ are membrane force and bending moment respectively and the matrices $\mathbf{A}, \mathbf{D}$ are the membrane and bending rigidities.

\section{ISOGEOMETRIC FORMULATION}

\subsection{Kinematics and displacement field}

The geometry and displacement field can be defined in the following form:

$$
\mathbf{x}=\sum_{a=1}^{n \times m} R_{a} \mathbf{x}^{a} ; \quad \mathbf{u}=\sum_{a=1}^{n \times m} R_{a} \mathbf{u}^{a}
$$

where $R_{a}$ is the NURBS basis function associated with the control point $a, \boldsymbol{x}$ is the position vector of the shell and the displacement vector $\mathbf{u}^{a}$ has three components such as

$$
\mathbf{u}^{a}=\left\{\bar{u}_{1}^{a}, \bar{u}_{2}^{a}, \bar{u}_{3}^{a}\right\}
$$

Note that the one-dimensional index $a$ as a pointer can be expressed as $a=m(i-1)+j$ associated with two-dimensional expression and therefore the $R_{a}$ of (30) can be interpreted as $R_{i, j}^{p, q}$ of $(6)$.

\subsection{Strain-displacement relationship matrix}

Using (30), the strains of (18) and (19) can be rewritten in the form of the strain-displacement relation matrix $\mathbf{B}$ as follows

$$
\boldsymbol{\varepsilon}=\sum_{a=1}^{n \times m} \overline{\mathbf{B}}^{a} \mathbf{u}^{a} ; \boldsymbol{\kappa}=\sum_{a=1}^{n \times m} \widetilde{\mathbf{B}}^{a} \mathbf{u}^{a}
$$

where the sub-matrices of $\overline{\mathbf{B}}^{a}$ and $\widetilde{\mathbf{B}}^{a}$ are

$$
\begin{aligned}
\overline{\mathbf{B}}^{a}= & {\left[\begin{array}{lll}
\overline{\mathrm{B}}_{11}^{a} \cdot e_{1} & \overline{\mathrm{B}}_{11}^{a} \cdot e_{2} & \overline{\mathrm{B}}_{11}^{a} \cdot e_{3} \\
\overline{\mathrm{B}}_{22}^{a} \cdot e_{1} & \overline{\mathrm{B}}_{22}^{a} \cdot e_{2} & \overline{\mathrm{B}}_{22}^{a} \cdot e_{3} \\
\overline{\mathrm{B}}_{12}^{a} \cdot e_{1} & \overline{\mathrm{B}}_{12}^{a} \cdot e_{2} & \overline{\mathrm{B}}_{12}^{a} \cdot e_{3}
\end{array}\right] ; } \\
\widetilde{\mathbf{B}}^{a} & =\left[\begin{array}{lll}
\widetilde{\mathrm{B}}_{11}^{a} \cdot e_{1} & \widetilde{\mathrm{B}}_{11}^{a} \cdot e_{2} & \widetilde{\mathrm{B}}_{11}^{a} \cdot e_{3} \\
\widetilde{\mathrm{B}}_{22}^{a} \cdot e_{1} & \widetilde{\mathrm{B}}_{22}^{a} \cdot e_{2} & \widetilde{\mathrm{B}}_{22}^{a} \cdot e_{3} \\
\widetilde{\mathrm{B}}_{12}^{a} \cdot e_{1} & \widetilde{\mathrm{B}}_{12}^{a} \cdot e_{2} & \widetilde{\mathrm{B}}_{12}^{a} \cdot e_{3}
\end{array}\right]
\end{aligned}
$$

in which $\overline{\mathrm{B}}_{j k}^{a}$ and $\widetilde{\mathrm{B}}_{j k}^{a}$ are

$$
\begin{gathered}
\overline{\mathrm{B}}_{j k}^{a}=\left(\frac{\partial R_{a}}{\partial x_{k}} a_{j}+\frac{\partial R_{a}}{\partial x_{j}} a_{k}\right), \\
\widetilde{\mathrm{B}}_{j k}^{a}=R_{a, j k} a_{3}+\frac{1}{\sqrt{a}}\left(\frac{\partial R_{a}}{\partial x_{1}} \cdot\left(a_{j, k} \times a_{2}\right)+\frac{\partial R_{a}}{\partial x_{2}} \cdot\left(a_{1} \times a_{j, k}\right)\right) \\
+\frac{a_{3} \cdot a_{j, k}}{\sqrt{a}}\left(\frac{\partial R_{a}}{\partial x_{1}} \cdot\left(a_{2} \times a_{3}\right)+\frac{\partial R_{a}}{\partial x_{2}} \cdot\left(a_{3} \times a_{1}\right)\right)
\end{gathered}
$$

\subsection{Free vibration analysis}

In the absence of external load and damping effects, the dynamic equilibrium equation based on principle of virtual work can be written as

$$
\int_{\bar{\Omega}} \delta \varepsilon \mathbf{A} \varepsilon \mathrm{d} \bar{\Omega}+\int_{\bar{\Omega}} \delta \boldsymbol{\kappa} \mathbf{D} \boldsymbol{d} \bar{\Omega}=\int_{\Omega} \delta \mathbf{u} \rho \ddot{\mathbf{u}} \mathrm{d} \Omega
$$

where $\mathbf{u}$ is the displacement, $\ddot{\mathbf{u}}$ is the acceleration, $\rho$ is the density of material and the notation $\delta$ denotes that the terms are virtual.

The relevant derivation takes place in finite-dimensional subspace to turn the above virtual statement of the problem into a system of algebraic equations. In this study, the subspaces are defined by using the NURBS basis:

$$
\mathbf{u}=\sum_{\mathrm{a}=1}^{n \times m} R_{a} \mathbf{u}_{\mathrm{a}}, \quad \ddot{\mathbf{u}}=\sum_{\mathrm{a}=1}^{n \times m} R_{a} \ddot{\mathbf{u}}_{\mathrm{a}}
$$

where $n \times m$ is the total number of the control point in the discretized domain and the virtual terms associated with the displacement and acceleration are

$$
\delta \mathbf{u}=\sum_{\mathrm{a}=1}^{n \times m} R_{a} \delta \mathbf{u}_{\mathrm{a}}, \quad \delta \ddot{\mathbf{u}}=\sum_{\mathrm{a}=1}^{n \times m} R_{a} \delta \ddot{\mathbf{u}}_{\mathrm{a}} .
$$

Substituting (36) and (37) into (35) yields 


$$
\delta \mathbf{u}^{\mathrm{T}}[\mathbf{K u}-\mathbf{M} \ddot{\mathbf{u}}]=0 .
$$

Since the virtual displacement $\delta \mathbf{u}$ is arbitrary, the above equation may be written as

$$
\mathbf{K u}-\mathbf{M} \ddot{\mathbf{u}}=0 .
$$

A general solution of (39) may be written

$$
\mathbf{u}=\boldsymbol{\phi}_{k} \mathrm{e}^{i \omega_{k}}
$$

Substituting (40) into (39) yields

$$
\left[\mathbf{K}-\omega_{k}^{2} \mathbf{M}\right] \boldsymbol{\phi}_{k}=0
$$

where $\boldsymbol{\phi}_{k}$ is a set of displacement-type amplitude at the control points otherwise known as the mode vector. $\omega_{k}$ is the natural frequency associated with the $k^{\text {th }}$ mode and $\mathbf{K}$ and $\mathbf{M}$ are global stiffness and mass matrices which contain contributions from element stiffness and mass matrices.

The structural stiffness and mass matrices in (41) can be written as

$$
\begin{aligned}
\mathbf{K}=\mathbf{K}_{a b} & =\overline{\mathbf{K}}^{a b}+\widetilde{\mathbf{K}}^{a b} \\
& =\int_{\bar{\Omega}} \overline{\mathbf{B}}^{a} \mathbf{A} \overline{\mathbf{B}}^{b} d \bar{\Omega}+\int_{\bar{\Omega}} \widetilde{\mathbf{B}}^{a} \mathbf{D} \widetilde{\mathbf{B}}^{b} d \bar{\Omega}, \\
\mathbf{M}=\mathbf{M}^{a b} & =\int_{\Omega} \mathbf{R}_{a}^{T} \rho \mathrm{R}_{b} d \Omega .
\end{aligned}
$$

Let the span is assumed to be as an isogeometric element and then the above equation can be written in the knot coordinate system as follows

$$
\begin{aligned}
& \prod_{e=1}^{n_{e l}}\left[K^{a b}\right]^{(e)}=\prod_{\substack{e=1 \\
n_{e l}}}^{n_{e l}} \iint_{k_{e}}^{k_{e+1}}\left[\overline{\mathbf{B}}^{a} \mathbf{D} \overline{\mathbf{B}}^{b}\right] \operatorname{det}(\mathrm{J}) \mathrm{d} \xi \mathrm{d} \eta+ \\
& \prod_{\substack{e=1 \\
n_{e l}}}^{n_{e l}} \iint_{k_{e}}^{k_{e+1}}\left[\widetilde{\mathbf{B}}^{a} \mathbf{G} \widetilde{\mathbf{B}}^{b}\right] \operatorname{det}(\mathrm{J}) \mathrm{d} \xi \mathrm{d} \eta, \\
& \prod_{e=1}^{n_{e l}}\left[\mathrm{M}^{a b}\right]^{(e)}=\prod_{e=1}^{\substack{e_{n}=1 \\
n_{e l}}} \iint_{k_{e}}^{k_{e+1}}\left[\mathrm{R}_{a}^{T} \widehat{\mathbf{m}} \mathrm{R}_{b}\right] \operatorname{det}(\mathrm{J}) \mathrm{d} \xi \mathrm{d} \eta
\end{aligned}
$$

where $\prod$ is the FE assembly operator (Hughes, 1987), $n_{e l}\left(=n_{\text {span }}\right.$ $\left.\times m_{\text {span }}\right)$ is the number of element, $\left[k_{e} k_{e+1}\right]$ is knot interval for integration in the $\xi$ and $\eta$, J is Jacobian matrix between $\boldsymbol{x}$ and $\xi, a, b$ is the basis function number associated with the target element and the matrix $\widehat{\mathbf{m}}$ is

$$
\widehat{\mathbf{m}}=\rho \cdot\left[\begin{array}{lll}
h & 0 & 0 \\
0 & h & 0 \\
0 & 0 & h
\end{array}\right]
$$

\section{NUMERICAL EXAMPLES}

In this section, we analyze several thin plates and shells by using the isogeometric thin shell element. The lowest frequencies of shells are calculated and the results are compared to the existing reference solutions (Kanok-nukulchai, 1979; Lee and Han, 2001; Lee et al., 1981; Leissa, 1969; Liew, 1992; Huang and Hinton, 1986).

\subsection{Circular plate}

A circular plate with clamped boundaries is analyzed. The thickness-span ratios $h / 2 r=0.01$ is used in this study. For analysis, the entire plate is discretized with meshes of 64 and 256 isogeometric elements illustrated in Figure 2 and three orders of basis function $\mathrm{p}=\mathrm{q}=3,4,5$ are used. Note that the control points may not be laid in the physical domain of structure since the present isogeometric approach uses NURBS definition for representing the geometry of structures. Therefore, the control points, which influence the essential boundary condition, are selectively fixed.

The resulting frequencies are presented in the dimensionless form:

$$
\lambda_{n}=w_{n} r^{2}\left(\frac{\rho}{D}\right)^{\frac{1}{2}}
$$

where $r$ is the radius of the circular plate, $\rho$ is the density of the material and the $D=E h^{3} / 12\left(1-v^{2}\right)$ is the flexural rigidity of the plate in which $\mathrm{E}$ is the elastic modulus and $v=0.3$ is the Poisson ratio.

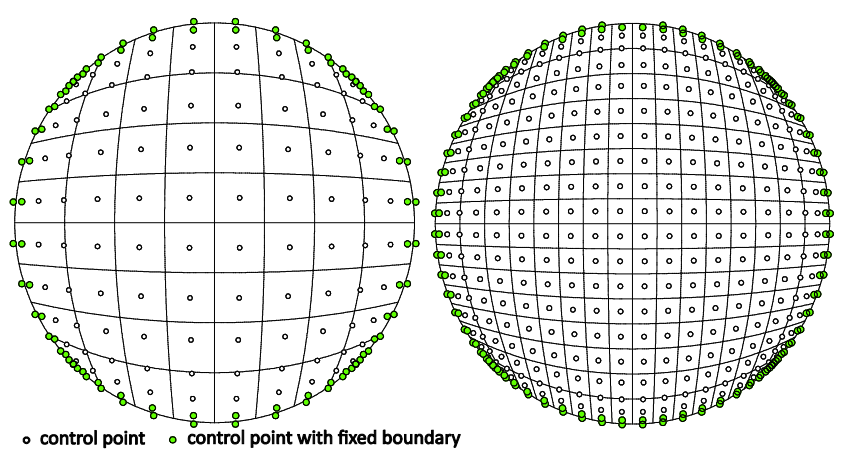

Figure 2. Mesh for a clamped circular plate $(\mathrm{p}=\mathrm{q}=5)$ : (left) 64 elements and (right) 256 elements

From numerical test, asymmetric and axisymmetric vibration modes are detected in this test. In the axisymmetric modes, multiple frequencies are obtained. The frequencies obtained from the present shell elements show a very good agreement with the exact solutions in overall modes with rather coarse mesh of 64 isogeometric elements. We illustrate the first fifteen mode shapes in Figure 3. The natural frequencies are calculated in the dimensionless form of (47) and provided in Table 1. 


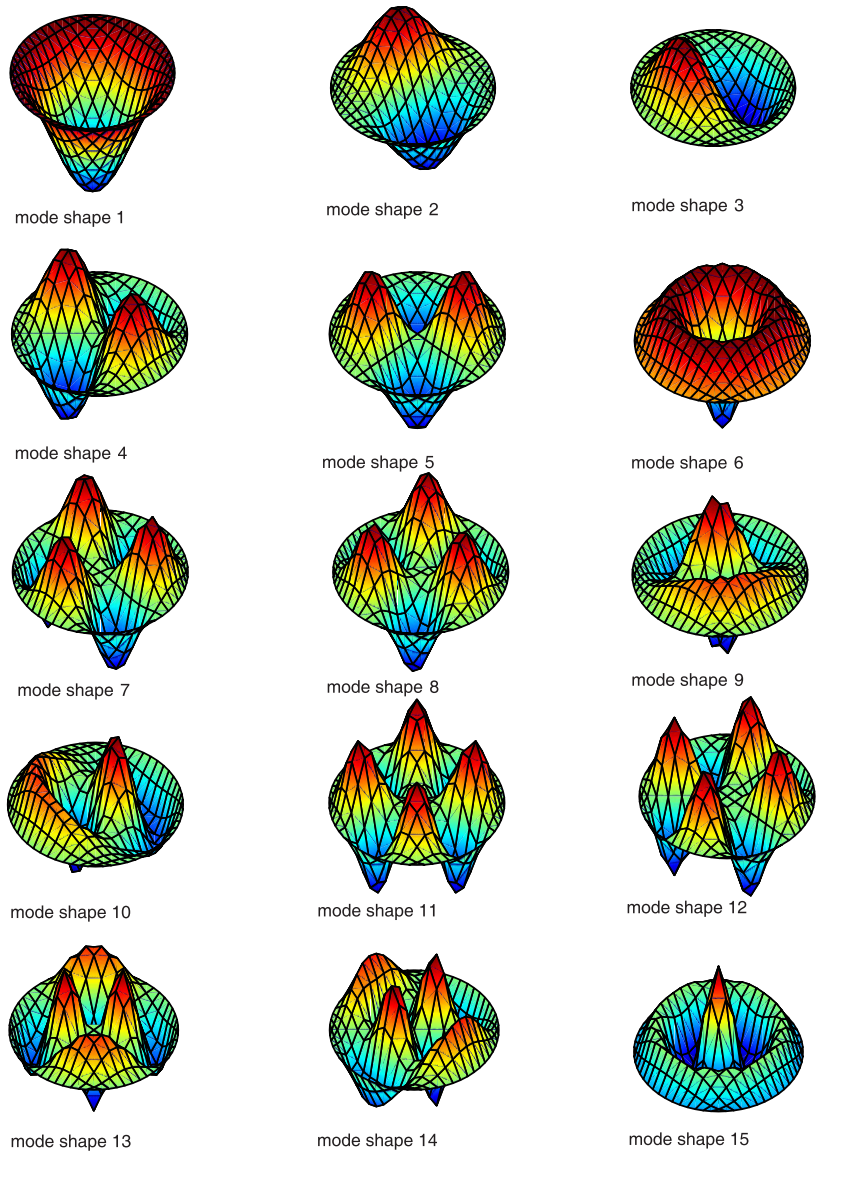

Figure 3. Mode shapes of clamped circular plate $(\mathrm{p}=\mathrm{q}=5$ and $\mathrm{h} / \mathrm{a}=0.01)$

Table 1. The non-dimensionalized natural frequencies $\lambda_{i}$ of a clamped circular plate with $\mathrm{h} / 2 \mathrm{r}=0.01$ and 64 elements.

\begin{tabular}{ccccccc}
\hline \multirow{2}{*}{ Mode } & \multicolumn{2}{l}{ Present : order of basis function } & & & \\
\cline { 2 - 4 } & $\mathrm{p}=\mathrm{q}=3$ & $\mathrm{p}=\mathrm{q}=4$ & $\mathrm{p}=\mathrm{q}=5$ & & & \\
& 10.216 & 10.216 & 10.216 & 10.213 & 10.214 & 10.216 \\
2 & 21.260 & 21.260 & 21.260 & 21.231 & 21.234 & 21.260 \\
- & 21.260 & 21.260 & 21.260 & - & - & - \\
3 & 34.877 & 34.877 & 34.877 & 34.782 & 34.793 & 34.877 \\
- & 34.877 & 34.877 & 34.877 & 34.792 & 34.803 & - \\
4 & 39.771 & 39.771 & 39.771 & 39.677 & 39.680 & 39.771 \\
5 & 51.030 & 51.030 & 51.030 & 50.835 & 50.859 & 51.030 \\
- & 51.030 & 51.030 & 51.030 & - & - & - \\
6 & 60.829 & 60.829 & 60.829 & 60.676 & 60.680 & 60.829 \\
- & 60.829 & 60.829 & 60.829 & - & - & - \\
7 & 69.667 & 69.666 & 69.666 & 69.303 & 69.359 & 69.666 \\
- & 69.667 & 69.666 & 69.666 & 69.338 & 69.364 & - \\
8 & 84.584 & 84.583 & 84.583 & 84.300 & 84.320 & 84.583 \\
- & 84.586 & 84.583 & 84.583 & 84.385 & 84.380 & - \\
9 & 89.107 & 89.104 & 89.104 & 88.985 & 88.975 & 89.104 \\
10 & 90.742 & 90.739 & 90.739 & 90.208 & 90.260 & 90.739 \\
- & 90.742 & 90.739 & 90.739 & - & - & - \\
\hline$N$
\end{tabular}

Note: Ref1: assumed natural strain shell element (Lee, 2001); Ref2: ninenode shell element (Kanok-nukulchai, 1979); Ref3: analytical solutions (Leissa, 1969).

\subsection{Elliptical plate}

The elliptical plate is analyzed. The geometry of the plate is presented in Figure 4.

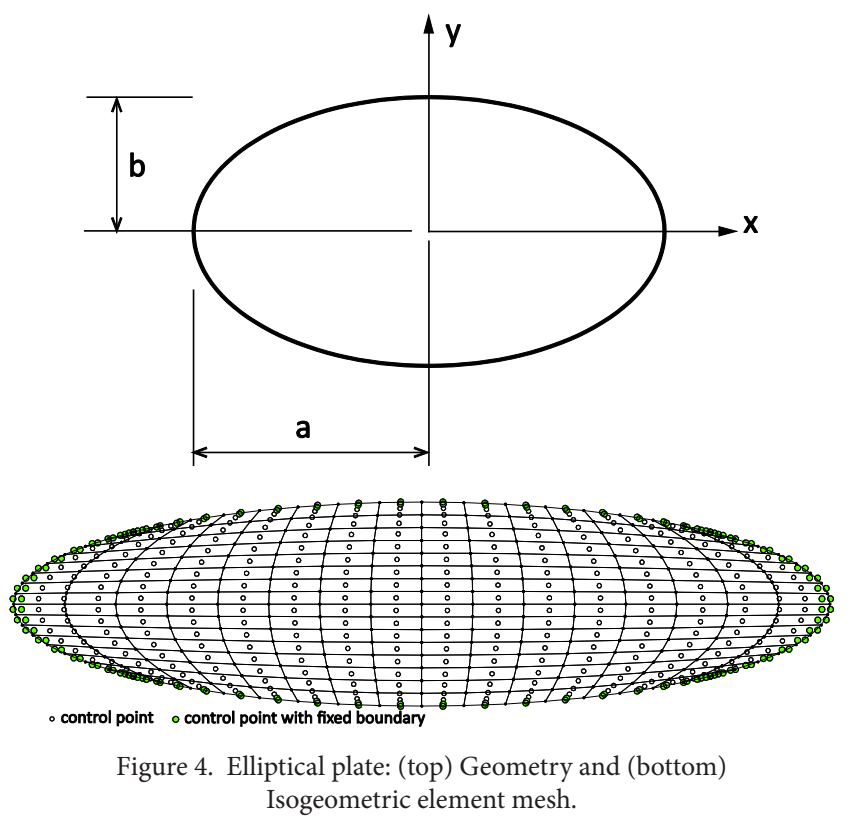

The plate has the aspect ratios $\mathrm{a} / \mathrm{b}=4$ and the thickness-span ratio is taken as $2 \mathrm{~h} / \mathrm{b}=0.01$. For analysis, 256 isogeometric elements are used. All units are assumed to be consistent.

The results are presented in the dimensionless form

$$
\lambda_{n}=w_{n} a^{2}\left(\frac{\rho}{D}\right)^{\frac{1}{2}}
$$

where $a$ is the radius of the elliptical plates in the $x$ direction, $\rho$ is the density of the material and the $D=E h^{3} / 12\left(1-v^{2}\right)$ is the flexural rigidity of the plate in which is the elastic modulus and $v=0.3$ is the Poisson ratio.

The present solutions are compared with the reference solution produced by Liew (1992) and the FE solutions obtained by using the shell element S9R5 of the ABAQUS and assumed natural strained shell element (Lee, 2001). The natural frequencies and mode shapes of the elliptical plates are presented in Figure 5 and Table 2 respectively.
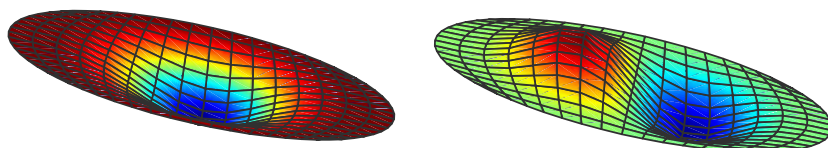

mode shape

mode shape 2

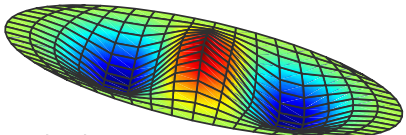

mode shape 3
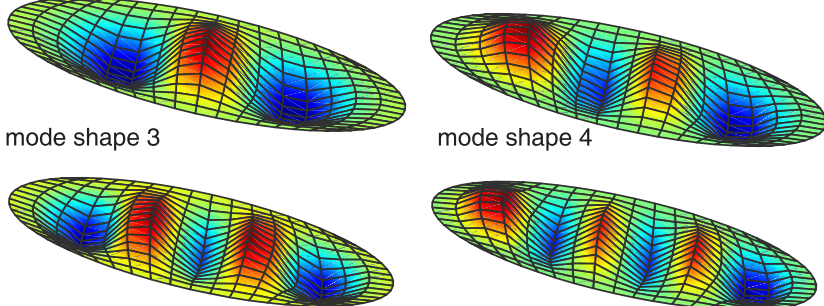

mode shape 5

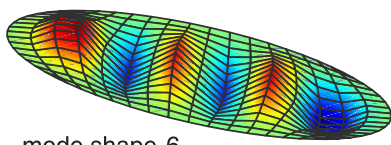

mode shape 6 


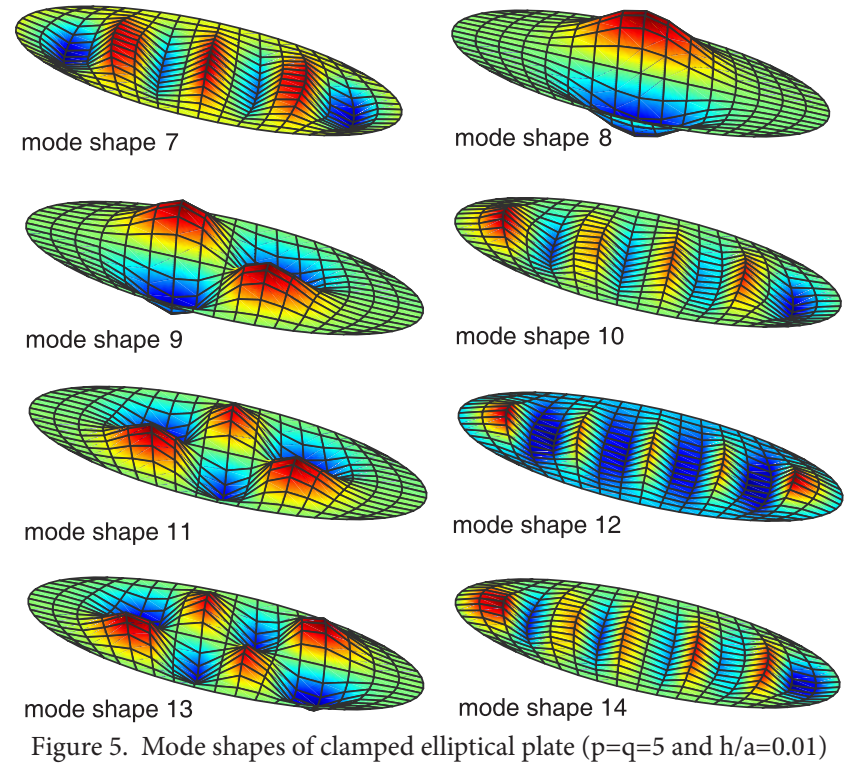

From Table 2, it is found to be that the present solutions have an excellent agreement with theoretical solutions. But there are some discrepancies in higher modes with FE solutions. Mostly, two FE reference solutions produces lower natural frequencies compare to theoretical solution (Liew, 1992) and the present isogeometric solutions.

Table 2. The non-dimensionalized natural frequencies $\lambda_{i}$ of clamped elliptical plate with $\mathrm{a} / \mathrm{b}=4$.

\begin{tabular}{|c|c|c|c|c|c|c|}
\hline \multirow{2}{*}{ Mode } & \multicolumn{3}{|c|}{ Present : order of basis function } & \multirow{2}{*}{ Ref1 } & \multirow{2}{*}{ Ref2 } & \multirow{2}{*}{ Ref3 } \\
\hline & $\mathrm{p}=\mathrm{q}=3$ & $\mathrm{p}=\mathrm{q}=4$ & $\mathrm{p}=\mathrm{q}=5$ & & & \\
\hline 1 & 97.599 & 97.599 & 97.599 & 97.5984 & 95.749 & 96.356 \\
\hline 2 & 115.594 & 115.594 & 115.594 & 115.608 & 112.757 & 113.898 \\
\hline 3 & 137.224 & 137.223 & 137.223 & 137.269 & 133.500 & 134.939 \\
\hline 4 & 162.643 & 162.639 & 162.639 & 164.325 & 157.296 & 159.59 \\
\hline 5 & 192.036 & 192.020 & 192.019 & 195.340 & 184.776 & 187.987 \\
\hline 6 & 225.593 & 225.538 & 225.532 & 255.095 & 215.724 & 220.247 \\
\hline 7 & 261.303 & 261.303 & 261.303 & - & 250.668 & 254.045 \\
\hline 8 & 263.524 & 263.352 & 263.326 & - & 250.668 & 256.461 \\
\hline 9 & 292.151 & 292.151 & 292.151 & - & 278.112 & 283.232 \\
\hline 10 & 306.116 & 305.629 & 305.536 & - & 289.620 & 296.699 \\
\hline 11 & 326.304 & 326.301 & 326.301 & - & 310.332 & 315.447 \\
\hline 12 & 353.850 & 352.607 & 352.310 & - & 333.060 & 340.987 \\
\hline 13 & 363.893 & 363.879 & 363.877 & - & 343.824 & 350.723 \\
\hline 14 & 405.064 & 404.741 & 403.901 & - & 380.760 & 389.143 \\
\hline 15 & 407.599 & 405.008 & 405.000 & - & 381.084 & 389.349 \\
\hline
\end{tabular}

Note: Ref1: analytical solution (Liew, 1992); Ref2: FE solution using Abaqus; Ref3: assumed natural strain shell element (Lee and Han, 2001).

\subsection{Curved fan blade}

The vibration of curved fan blade has been frequently studied. Lee et al. (1981) investigated the behaviour of this structure using the Ritz method based on classical thin shallow shell theory in which the different polynomial terms are used. The shell geometry is presented in Figure 6.

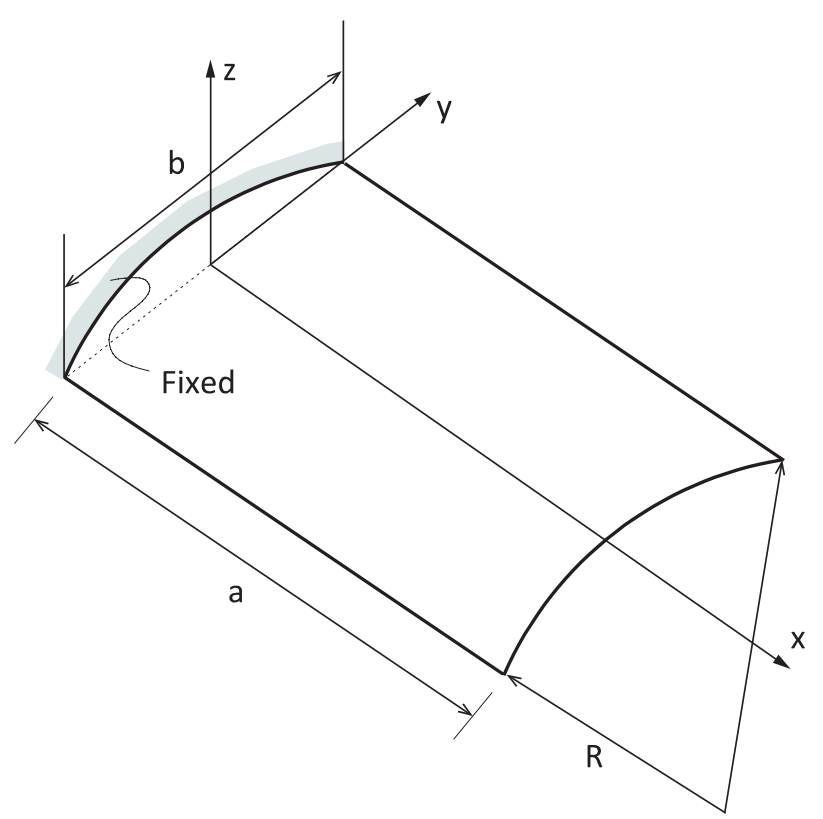

Figure 6. Geometry of curved fan blade

The aspect ratio $(\mathrm{a} / \mathrm{b}=5)$, shallowness ratio $(\mathrm{b} / \mathrm{R}=0.5)$ and widththickness ratio $(b / h=100)$ are used in the analysis. The initial location of control point with exact geometry of fan blade section is illustrated in Figure 7.

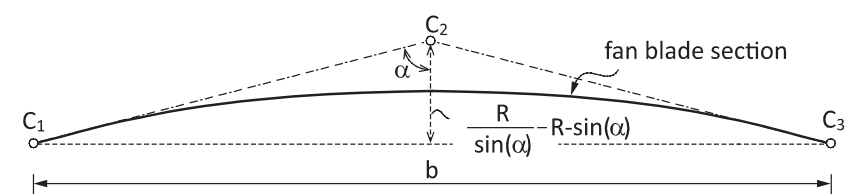

Figure 7. Initial location of control points for fan blade section

After 4 times of h-refinement with the orders of basis function $\mathrm{p}=\mathrm{q}=5$ in the direction of $x$ and $\mathrm{y}$ directions, isogeometric elements is produced and is used to analyze the entire shell.

The result is expressed in non-dimensional form:

$$
\lambda_{n}=w_{n} a b\left(\frac{\rho}{D}\right)^{\frac{1}{2}}
$$

where, the flexural rigidity is $D=E h^{3} / 12\left(1-v^{2}\right)$.

The results are compared with the solution obtained by the Ritz method where six and five polynomial terms are used in the $x$ and $y$ directions respectively. From the test, the natural frequencies and the mode shapes are presented in Table 3 and Figure 8.
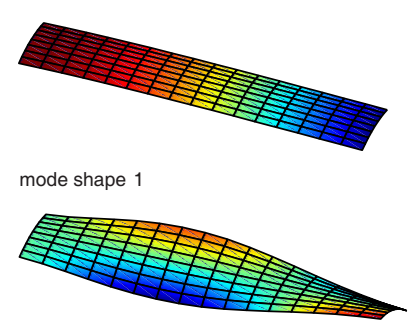

mode shape $\square$

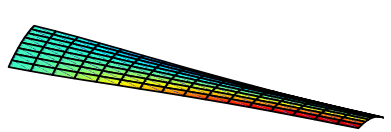

mode shape $\square$

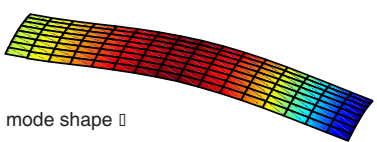




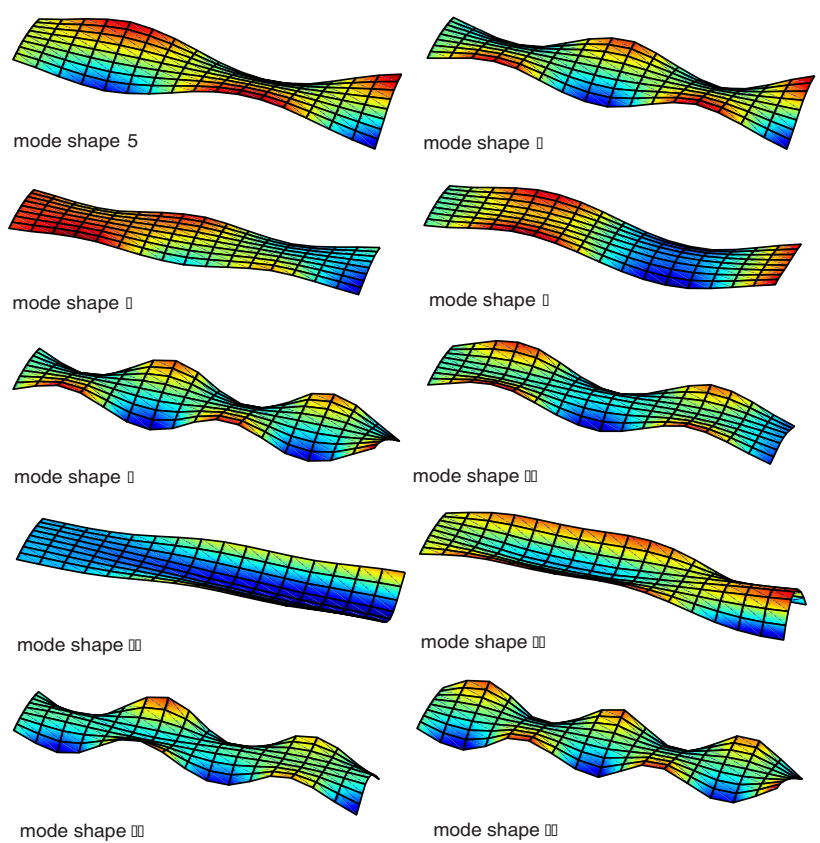

Figure 8. Mode shapes of a curved fan blade ( $\mathrm{p}=\mathrm{q}=5$ and $\mathrm{h} / \mathrm{a}=0.01$ )

Table 3. The non-dimensionalized natural frequencies $\lambda_{i}$ of a curved fan blade with the aspect ratio $\mathrm{a} / \mathrm{b}=5$.

\begin{tabular}{ccccccc}
\hline \multirow{2}{*}{ Mode } & \multicolumn{2}{c}{ Present : order of basis function } & & & \\
\cline { 2 - 4 } & $\mathrm{p}=\mathrm{q}=3$ & $\mathrm{p}=\mathrm{q}=4$ & $\mathrm{p}=\mathrm{q}=5$ & & & \\
& 4.378 & 4.377 & 4.377 & 4.322 & 4.441 & 4.437 \\
1 & 6.979 & 6.978 & 6.977 & 7.175 & 7.122 & 7.117 \\
2 & 22.286 & 22.282 & 22.280 & 22.899 & 22.797 & 22.774 \\
3 & 26.385 & 26.383 & 26.381 & 25.995 & 26.675 & 26.644 \\
4 & 41.326 & 41.318 & 41.314 & 42.394 & 42.409 & 42.340 \\
5 & 64.868 & 64.865 & 64.864 & 65.217 & 65.933 & 65.895 \\
6 & 65.603 & 65.587 & 65.579 & 65.281 & 66.570 & 66.478 \\
7 & 65.727 & 65.718 & 65.714 & 67.064 & 67.415 & 67.209 \\
8 & 94.679 & 94.656 & 94.645 & - & - & - \\
9 & 101.145 & 101.127 & 101.119 & - & - & - \\
10 & 108.147 & 108.146 & 108.145 & - & - & - \\
11 & 116.041 & 116.039 & 116.037 & - & - & - \\
12 & 128.262 & 128.228 & 128.213 & - & - & - \\
13 & 129.840 & 129.815 & 129.804 & - & - & - \\
14 & 138.339 & 138.335 & 138.333 & - & - & - \\
15 & 139 &
\end{tabular}

Note: Ref1: analytical solution (Lee et al., 1981); Ref2: nine-node shell element (Huang and Hinton, 1986); Ref3: assumed natural strain shell element (Lee and Han, 2001).

\section{CONCULSIONS}

A thin shell element is developed by using isogeometric concept and used to investigate the behaviours of thin plate and shell structures under free vibration. Numerical examples are carried out to demonstrate the efficiency and accuracy of the present isogeometric shell element. From numerical results, the present isogeometric shell element can produce reliable natural frequencies and associated mode shapes of shells with enough accuracy and it can be used to predict vibrational behaviours of thin plate and shell structures. There is also a possibility of using the present isogeometric shell element in the analysis of shells having arbitrary boundary shapes since the present shell element can represent exact geometry of structures using NURBS definition. Finally, the present isogeometric solutions described in this paper are provided as future reference solutions on the structural vibrations of thin plate and shell structures.

\section{REFERENCES}

ABAQUS manual, Theory and Users Manuals. Hibbit, Karlson and Sorensen, Inc., Version 5.7.

Cottrell, J.A., Bazilevs, Y. and Hughes, T.J.R. (2009). Isogeometric Analysis: Towards Integration of CAD and FEA. Wiley.

De Boor, C. (1978) A Practical Guide to Splines. Springer.

Flügge, W. (1934) Statik und Dynamik der Schalen. SpringerVerlag, Berlin, Germany.

Huang, H.C. and Hinton, E. (1986) A new nine node degenerated shell element with enhanced membrane and shear interpolation. Int. J. Num. Meth. Engng., 22, pp.73-92.

Hughes, T.J.R. (1987) The Finite Element Method- Linear Static and Dynamic Finite Element Analysis. New Jersey: Prentice-Hall.

Hughes, T.J.R., Cottrell, J. A. and Bazilevs, Y. (2005) Isogeometric analysis: CAD, finite elements, NURBS, exact geometry and mesh refinement. Comput. Methods Appl. Mech. Eng., 194(3941), pp.4135-4195.

Hughes, T.J.R. and Evans J.A. (2010). Isogeometric analysis. ICES Report 10-18, The Institute of Computational Engineering and Science, University of Texas Austin.

Kanok-nukulchai, W. (1979) A simple and efficient finite element for general shell element. Int. J. Num. Meth. Engng. 14, pp.179-200.

Lee, J.K., Leissa, A.W. and Wang, A.J. (1981) Vibrations of cantilevered shallow cylindrical shells of rectangular planform. J. Sound and Vibration, 78, pp.311-328.

Lee, S.J. and Kim, H.R. (2012) Vibration and buckling of thick plates using isogeometric approach. Architectural Research, 15, pp. 35-42.

Lee, S.J. and Han, S.E. (2001) Free-vibration analysis of plates and shells with a nine-node assumed natural degenerated shell element. Journal of Sound and Vibration, 241, pp.605-633.

Lee, S.J. and Park, K.S. (2013) Vibrations of Timoshenko beams with isogeometric approach. Applied Mathematical Modelling. 37(22), pp.9174-9190.

Leissa, A.W. (1969) Vibrations of plates. NASA SP-160, Washington D.C. Leissa, A.W. (1973) Vibrations of shells. NASA SP-288, Washington D.C.

Liew, K.M. (1992) Use of two-dimensional orthogonal polynomials for vibration analysis of circular and elliptical plates. J. Sound and Vibration, 154, pp.261-269.

Liew, K.M. (1995) Research on thick plate vibration: a literature survey. J. Sound and Vibration, 180, pp.163-176.

Love, A.E.H. (1888) The small free vibrations and deformation of a thin elastic shell, Philosophical transactions of royal society of London A, 179, pp.491-549.

Qatu, M.S. (1992) Review of shallow shell vibration research, Shock Vibration Digest, 24, pp.3-15.

Rayleigh, J.W.S. (1894), Theory of sound. MacMillan Inc., London, England

(Received February 5, 2014/Accepted May 7, 2014) 